\title{
Incremental prognostic value of stress echocardiography as an adjunct to exercise electrocardiography after uncomplicated myocardial infarction
}

\author{
R Bigi, A Desideri, A Galati, J J Bax, C Coletta, C Fiorentini, P M Fioretti
}

\begin{abstract}
Objective-To assess the prognostic value of stress echocardiography as an adjunct to exercise electrocardiography in patients with uncomplicated acute myocardial infarction.

Design-496 patients underwent a maximum exercise ECG and pharmacological stress echocardiography (406 dobutamine and 90 dipyridamole) within 15 days of uncomplicated acute myocardial infarction and were followed for a mean of 25 months (range 1-74 months) for reinfarction, unstable angina, and cardiac death. Patients undergoing revascularisation were omitted.

Results-Exercise ECG was positive in 162 patients (32.6\%) and low threshold positive $(<100 \mathrm{~W})$ in $91(18 \%)$. Stress echocardiography was positive in 239 patients $(48 \%)$ (194 with dobutamine and 45 with dipyridamole stress). The agreement between the two tests was $63 \%$ $(\kappa=0.24,95 \%$ confidence interval 0.15 to 0.33 ). Sixty nine spontaneous events occurred (14 cardiac deaths, 26 reinfarctions, and 29 with unstable angina requiring hospital admission), and 126 patients underwent revascularisation (39 coronary angioplasty and 87 bypass surgery). By receiver operating characteristic curve analysis, stress echocardiography provided incremental prognostic information compared with clinical data. A low threshold positive exercise ECG was associated with a worse outcome, but there was a fivefold increase in risk in patients with positive stress echocardiography who also had a high threshold (>100 W) positive exercise ECG. Eventfree survival of patients with both tests positive was significantly less than in patients with only one positive test or with both tests negative.

Conclusions-Stress echocardiography provides additional prognostic information after uncomplicated acute myocardial infarction, but the greatest gain is found in patients with a high threshold positive exercise ECG.
\end{abstract}

(Heart 2001;85:417-423)

Keywords: risk stratification; myocardial infarction; stress echocardiography; exercise stress testing

Cardiovascular

Research Foundation,

Castelfranco Veneto,

Italy

R Bigi

A Desideri

A Galati

C Coletta

Department of Cardiology, University

Hospital, Leiden, Netherlands

J J Bax

Division of Cardiology, $S$ Paolo Hospital and University of Milan, Italy

C Fiorentini

IRCAB Foundation and Cardiology

Institute, $\mathbf{S}$ Maria della Misericordia Hospital,

Udine, Italy

P M Fioretti

Correspondence to:

Dr Riccardo Bigi, Via Visoli

1, 23037 Tirano (SO), Italy

rbigi@tiscalinet.it

Accepted 25 October 2000
Patients with uncomplicated acute myocardial infarction generally have less extensive coronary artery disease, preserved left ventricular function, and a favourable prognosis. ${ }^{1} \mathrm{Al}-$ though the link between residual ischaemia and subsequent coronary events is questionable in these patients, a weak but significant correlation was found in the large population of the GISSI 2 study (gruppo Italiano per lo studio della sopravvivenza nell'infarto miocardico). ${ }^{2}$ Furthermore, the DANAMI study (Danish multicentre randomised study of invasive versus conservative treatment in patients with inducible ischaemia after thrombolysis in acute myocardial infarction) ${ }^{3}$ has recently shown that invasive treatment of patients with inducible ischaemia after an infarct results in a reduction in the incidence of reinfarction and fewer admissions for unstable angina. On the other hand, routine early invasive management consisting of coronary angiography and revascularisation failed to show a prognostic benefit over a conservative, ischaemia guided approach in non-Q wave myocardial infarction in the VANQWISH study (Veterans Affairs non-Q-wave infarction strategies in hospital). ${ }^{4}$ Therefore a predischarge stratification of risk including the assessment of inducible ischaemia seems to be justified in patients with uncomplicated myocardial infarction despite their low pretest likelihood of further events.

Detection of inducible ischaemia traditionally relies on an exercise ECG, but the inability to achieve an adequate level of exercise and the presence of resting ECG abnormalities are common limitations of this technique. In an attempt to overcome these limitations, imaging techniques have increasingly been used; in particular, stress echocardiography has emerged as a safe and cost-effective tool in the postinfarction setting. ${ }^{5-8}$ However, its value as an additional test in assessing the prognosis of postinfarct patients who are able to exercise has not been adequately investigated, while data on the long term follow up of patients undergoing stress echocardiography for risk stratification after acute myocardial infarction are still lacking.

Our aim in this study was to assess prospectively the long term prognostic value of pharmacological stress echocardiography as an adjunct to predischarge exercise ECG in patients with uncomplicated acute myocardial infarction. 


\section{Methods}

STUDY POPULATION

The initial study population consisted of 675 consecutive patients with acute myocardial infarction eligible for symptom limited exercise electrocardiography before discharge from hospital. Acute myocardial infarction was documented by typical chest pain lasting for more than 30 minutes, characteristic ECG changes, and raised creatine kinase and CK-MB isoenzyme fraction. Left ventricular ejection fraction was measured by cross sectional echocardiography using Simpson's rule.

Exclusion criteria were as follows:

- Age $>75$ years (25 patients).

- Features of complicated infarction (overt heart failure, complex arrhythmias, and early recurrence of myocardial ischaemia) (35 patients).

- Previous acute myocardial infarction (45 patients).

- Ejection fraction $<40 \%$ (48 patients).

- ECG abnormalities which would preclude the reading of an exercise ECG (such as left ventricular hypertrophy, left bundle branch block, ventricular pre-excitation, or digoxin use) (20 patients).

- Technically inadequate echocardiographic window (6 patients).

After these exclusions, 496 patients entered the study, which was prospective. These patients underwent an exercise ECG and pharmacological stress echocardiography (406 dobutamine; 90 dipyridamole) in random order within 14 days of the acute event, after cardioactive drug washout of at least five half lives. They were then followed up for a mean period of 25 months (range 1-72 months). Age, sex, infarct location, diabetes, hypertension, and smoking were taken into account as clinical variables.

The pretest probability of subsequent cardiac events was calculated using the risk chart of GISSI-Prevenzione trial. ${ }^{9}$ This large multicentre study involving 11324 patients provides a probability estimate of relevant cardiac events (non-fatal reinfarction and death) in the 18 months following hospital discharge in relation to age, sex, risk factors, and hospital course.

Patients were referred for coronary angiography at the discretion of the staff cardiologist. The decision to perform revascularisation procedures was made by the clinical team without knowledge of the aim of the study.

\section{EXERCISE STRESS TESTING}

Patients performed symptom limited bicycle exercise ECG test (25 W incremental loading every two minutes) 6-12 days after the infarction. The 12 lead ECG was monitored continuously throughout the test for rhythm, rate, and ST-T wave changes by means of the computer assisted Marquette Case 15 system (Marquette Electronics Inc, Milwaukee, Wisconsin, USA). The occurrence of significant anginal pain, ventricular tachycardia, major conduction abnormalities, ST depression of more than $3 \mathrm{~mm}$, limiting symptoms (such as dyspnoea, dizziness, leg fatigue, and so on), or an excessive rise (above $230 \mathrm{~mm} \mathrm{Hg}$ ) or fall $(\geqslant 30 \mathrm{~mm} \mathrm{Hg}$ ) in systolic blood pressure were regarded as interruption criteria.

Both ST depression in one or more leads, excluding aVR and V1, and ST elevation in leads without pathological $Q$ waves were considered. Horizontal or downsloping ST depression of $>1 \mathrm{~mm}$ measured $80 \mathrm{~ms}$ after the J point, and ST elevation of $>1 \mathrm{~mm}$ measured $40 \mathrm{~ms}$ after the J point, were regarded as positive results. The positivity was defined as "low threshold" if it occurred at a workload of $<100 \mathrm{~W}$.

STRESS ECHOCARDIOGRAPHY

Stress echocardiography was performed 7-14 days after the infarction using Acuson XP-10 (Acuson Corp, Mountain View, California, USA) or Hewlett-Packard Sonos 2500 echo equipment (Hewlett-Packard Inc, Andover, Massachusetts, USA). During the test, the 12 lead ECG and cross sectional echocardiogram were monitored continuously. A 12 lead ECG was recorded each minute and blood pressure was taken at the end of each infusion stage. A dobutamine protocol (406 patients) and a dipyridamole protocol (90 patients) were both used. Dobutamine was infused according to a protocol based on three minute stages of 5, 10, 20,30 , and $40 \mu \mathrm{g} / \mathrm{kg} / \mathrm{min}$, plus additional atropine $(0.25-1 \mathrm{mg})$ if required to achieve $85 \%$ of the age predicted maximum heart rate. Metoprolol and sublingual nitrates were available as antagonists. Dipyridamole $(0.56 \mathrm{mg} / \mathrm{kg})$ was infused over four minutes; a second dose of $0.28 \mathrm{mg} / \mathrm{kg}$ in two minutes was added after four minutes if the response was inadequate, up to a cumulative final dose of $0.84 \mathrm{mg} / \mathrm{kg}$. Aminophylline (70-240 mg given over one to three minutes) was used as an antidote.

Evident deterioration of the wall motion pattern as well as ST depression of $>3 \mathrm{~mm}$, ST elevation of $>1 \mathrm{~mm}$ in remote leads, severe chest pain, significant arrhythmias, and a decrease in blood pressure of $>30 \mathrm{~mm} \mathrm{Hg}$ were considered reasons for terminating the tests.

Standard apical and parasternal views were recorded in a cine loop quad screen format on super VHS videotape in order to achieve optimal comparison of rest and stress images. Computer assisted analysis of images was used to improve the accuracy of interpretation and reduce intra- and interobserver variability. The examining cardiologists were all skilled in the technique and had passed a quality control assessment as previously described. ${ }^{6}$ All echocardiograms were analysed by two experienced observers whose agreement on the interpretation was $94 \%$. In case of disagreement, a third observer reviewed the images without knowledge of the previous assessments and a majority decision was achieved. The readers were blinded to the exercise ECG results.

For the semiquantitative assessment, the left ventricular wall was divided into 16 segments ${ }^{10}$ and scored using a four point scale: $1=$ normal; 2 = hypokinetic; $3=$ akinetic; $4=$ dyskinetic. A wall motion score index (WMSI) was 
calculated by adding the numerical value assigned to each segment and dividing by the number of visualised segments. An inducible wall motion abnormality was defined as worsening of wall motion in at least two segments as compared with rest or low dose. We considered worsening of wall motion to be a change from normal wall thickening to hypokinesis, akinesis, or dyskinesis, and from hypokinesis to akinesis or dyskinesis, but not from akinesis to dyskinesis. ${ }^{11}$ The test was considered positive if there was deterioration of wall motion in dysfunctional segments, or if new wall motion abnormalities developed in normokinetic regions. A biphasic response to dobutamine administration (that is, low dose wall motion improvement followed by high dose deterioration) was also considered as a criterion of positivity. The test was defined as negative if there was no evident change or if development of hyperkinetic wall motion was observed.

FOLLOW UP

Outcome was determined from interviews with the patients in the outpatient clinic, from hospital case notes, and from telephone interviews with the patients, close relatives, or referring physicians. Only spontaneous events (cardiac death, non-fatal reinfarction, and unstable angina requiring hospital admission) were taken into account. Death was defined as cardiac if strictly related to proved cardiac causes (such as fatal reinfarction, acute heart failure, or malignant arrhythmias); reinfarction was diagnosed on the basis of documented ECG changes and of typical cardiac enzyme release; and unstable angina was defined as "crescendo angina" and rest or minimal effort angina in the absence of clear cut ECG and cardiac enzyme changes indicating myocardial infarction.

All subjects undergoing revascularisation were censored at the time of the procedure, because it was assumed that it would cause underestimation of the spontaneous event rate. Only the first event was taken into account for statistical analysis, so there was no overlap between the two groups.

STATISTICAL ANALYSIS

Data are expressed as mean (SD). The 95\% confidence interval (CI) is given when appropriate. Continuous variables were compared using Student's $t$ test. The Kruskal-Wallis one way analysis of variance on ranks was used for multiple comparison in case of non-normal distribution. The Kolmogorov-Smirnov test was used to assess normality. Categorical variables were compared by $\chi^{2}$ statistics. The $\kappa$ value was used to assess the agreement between exercise ECG and stress echocardiography results.

Significance was set at a probability value of $p<0.05$. The post-test probability of cardiac events was calculated by means of the Bayes's theorem of conditional probability using the following formula:

post-test odds $=$ pretest odds $\times$ likelihood ratio, where odds $=$ probability $/(1-$ probability $)$, and likelihood ratio $=$ sensitivity $/(1-$ specificity) in case of a positive result or (1 sensitivity)/specificity in the case of a negative result.

Conversion to post-test probability was derived as: (post-test odds) $/(1+$ post-test odds).

The probability of subsequent cardiac events was calculated according to a sequential model including clinical data first, clinical data plus exercise ECG result second, and clinical data plus exercise ECG plus stress echocardiography result last. The postexercise ECG probability was considered as the pretest probability for stress echocardiography.

Receiver operating characteristic (ROC) curves were generated for each step of the model and comparisons were made using the area under the ROC curve method. ${ }^{12}$

The log-rank test was used to compare Kaplan-Meier event-free survival curves. The SPSS statistical package (release 7.5.1, 1996 for Windows) was used.

\section{Results}

PATIENT DATA

The study population was predominantly male (417/496) with a mean (SD) age of 58 (9) years. The infarct was anterior in 158 , inferior in 257, and non-Q wave in 81 patients; thrombolytic treatment was given in 267 cases (54\%). Peak creatine kinase was 2012 (1879) $\mathrm{IU} / \mathrm{ml}$ (normal < $250 \mathrm{IU} / \mathrm{ml}$ ). The clinical characteristics of patients with and without spontaneous cardiac events were similar (table 1).

\section{STRESS TEST DATA}

No life threatening complication occurred during either exercise ECG or pharmacological stress echocardiography.

Exercise ECG was positive in 162 patients $(33 \%)$ and low threshold positive in $91(18 \%)$. Maximum achieved workload and ratepressure product were, respectively, 96 (30) W and 23856 (5996). Primary reasons for exercise test termination were muscular fatigue in 240 , exhaustion in 118 , chest pain in 39 , excessive increase in blood pressure in 10, and ECG changes in 89.

Stress echocardiography was positive in 239 patients (48\%) (194 undergoing dobutamine stress and 45 dipyridamole stress). High dose dipyridamole $(0.84 \mathrm{mg} / \mathrm{kg})$ was given in 78 of the 90 patients in this group. In the

Table 1 Clinical characteristics of patients with and without spontaneous events

\begin{tabular}{llll}
\hline & $\begin{array}{l}\text { No events } \\
(n=427)\end{array}$ & $\begin{array}{l}\text { Events } \\
(n=69)\end{array}$ & p Value \\
\hline Age (years) (mean (SD)) & $57.9(9.2)$ & $58.8(9.8)$ & NS \\
Female & $68(16 \%)$ & $11(16 \%)$ & NS \\
Non-Q wave MI & $66(15 \%)$ & $15(21 \%)$ & NS \\
Thrombolysis & $228(53 \%)$ & $39(56 \%)$ & NS \\
Diabetes & $51(12 \%)$ & $13(19 \%)$ & NS \\
Smoking & $262(61 \%)$ & $41(59 \%)$ & NS \\
Hypertension & $88(20 \%)$ & $18(26 \%)$ & NS \\
WMSI at rest (mean (SD)) & $1.4(0.3)$ & $1.4(0.3)$ & NS \\
\hline
\end{tabular}

Values are $\mathrm{n}(\%)$ unless specified.

MI, myocardial infarction; WMSI, wall motion score index. 
Table 2 Relative risk of exercise ECG (ExT) with 95\% confidence intervals (CI)

\begin{tabular}{lll}
\hline & $\begin{array}{l}\text { Risk } \\
\text { ratio }\end{array}$ & $95 \%$ CI \\
\hline Negative ExT & 0.9 & $(0.6$ to 1.4$)$ \\
High threshold positive ExT & 0.9 & $(0.3$ to 1.6$)$ \\
Low threshold positive ExT & 1.4 & $(0.9$ to 1.8$)$ \\
\hline
\end{tabular}

dobutamine group, the maximum dobutamine dose was $36(6) \mu \mathrm{g} / \mathrm{kg} / \mathrm{min}$, and additional atropine was necessary in 166 of the 406 patients. Test end points were peak heart rate or maximum drug dose in 207 , chest pain in 49 , significant arrhythmias in 12 , side effects (paraesthesiae, nausea, or palpitations) in 11, ECG changes in four, and wall motion abnormalities in 213.

Exercise ECG and stress echocardiography gave a concordant result in 311 of 496 cases (63\%) ( $\kappa$ value $0.24,95 \%$ CI 0.16 to 0.33 ).

FOLLOW UP DATA

Mean follow up duration was 25 months (range 1-74 months). Data were available for all patients. In all, 198 events occurred after hospital discharge: 126 revascularisation procedures (39 coronary angioplasties and 87 bypass grafts), 69 spontaneous cardiac events (14 cardiac death, 26 reinfarction, and 29 unstable angina requiring hospital admission), and three non-cardiac deaths. The spontaneous event rate was similar among patients with a positive exercise ECG $(14.8 \%)$ and positive stress echocardiography (14.2\%).

Precipitating factors leading to revascularisation in patients without spontaneous events were the recurrence of anginal symptoms resistant to full medical treatment, and the evidence of extensive exercise induced silent ischaemia.

OUTCOME PREDICTION

According to the GISSI-Prevenzione risk chart, the mean pretest probability of cardiac events in the study group was $5.3(2.4) \%$ (range 2.2-

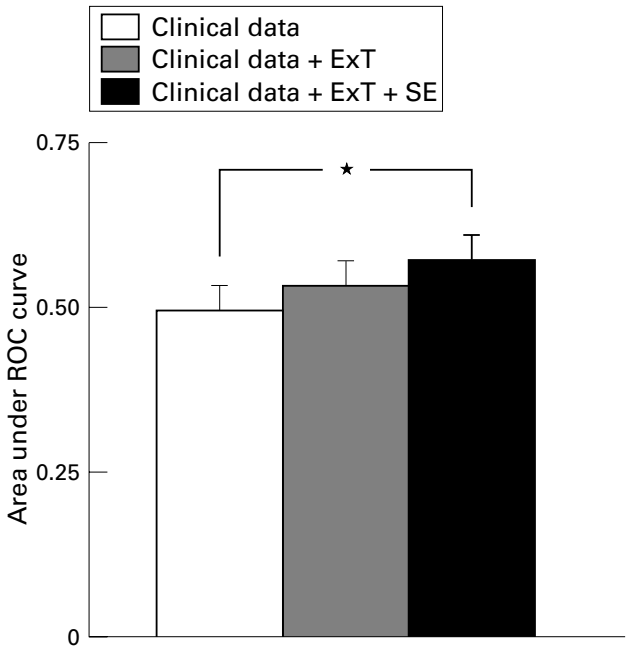

Figure 1 Comparison of the individual prognostic models by area under receiver operating characteristic (ROC) curve method (see text for explanation). Areas are shown with their $95 \%$ confidence intervals; ${ }^{\star} p<0.05$. ExtT, exercise ECG; SE, stress echocardiography.
Table 3 Relative risk of stress echocardiography (SE) according to the exercise ECG (ExT) results

\begin{tabular}{lll}
\hline & Negative SE & Positive SE \\
\hline Negative ExT & $0.9(0.4$ to 1.5$)$ & $1.1(0.5$ to 1.6$)$ \\
High threshold positive ExT & $1.0(0.4$ to 2.8$)$ & $5.2(0.6$ to 41$)$ \\
Low threshold positive ExT & $1.1(0.3$ to 2.6$)$ & $1.2(0.4$ to 3.2$)$ \\
\hline Values are means (95\% confidence intervals). &
\end{tabular}

$19.5 \%)$. Spontaneous events occurred in 24 patients with a positive and 45 with a negative exercise ECG; and in 34 patients with positive and 35 with negative stress echocardiography. The relative risk of negative and positive exercise ECG is given in table 2 .

Incremental ROC analysis of clinical data, clinical data plus exercise ECG, and clinical data plus exercise ECG plus stress echocardiography are summarised in fig 1 . The addition of stress echocardiography provided the most accurate outcome prediction, which was significantly superior to clinical data alone, while the addition of exercise ECG data alone did not improve the outcome prediction. However, the incremental prognostic value of stress echocardiography mainly came from the group of patients with high threshold positive exercise ECG results, in whom positive stress echocardiography was associated to a fivefold increase in risk of cardiac events. Conversely, no appreciable contribution of stress echocardiography was found in patients with low threshold positive or negative exercise ECG (table 3).

Event-free survival according to the exercise ECG result is shown in fig 2. Patients with exercise capacity less than $100 \mathrm{~W}$ did worse than the other groups independently of the result of the test, while patients with a low threshold positive test showed the lowest event-free survival. The
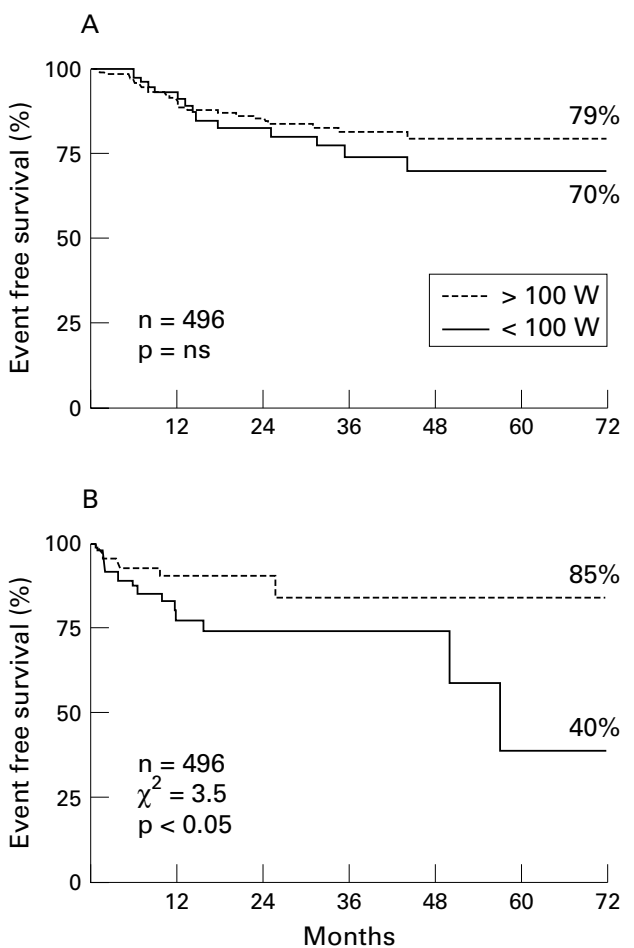

Figure 2 Kaplan-Meier event-free survival curves according to the workload achieved during negative $(A)$ and positive (B) exercise ECGs. 

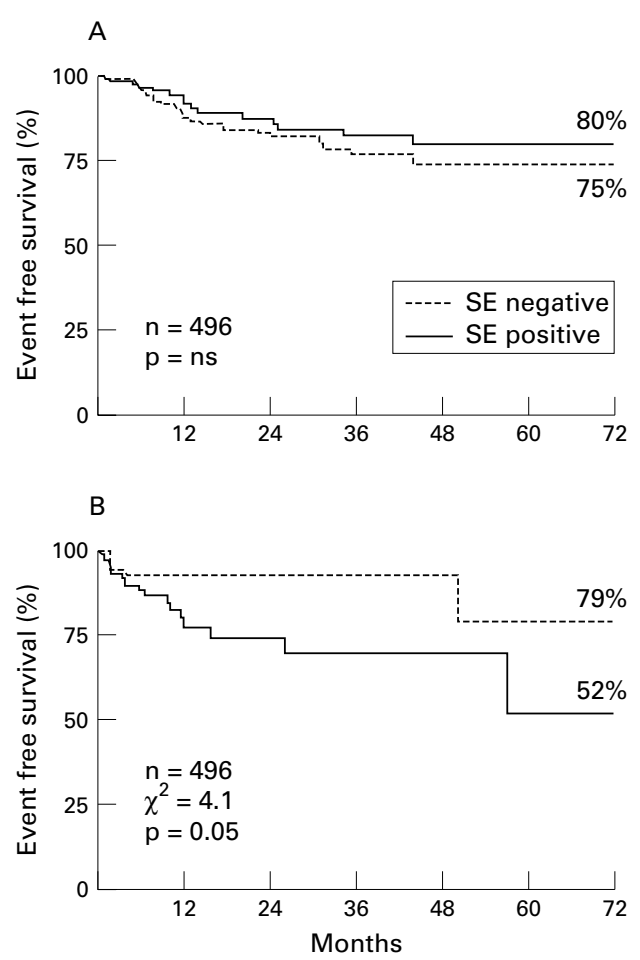

Figure 3 Kaplan-Meier event-free survival curves according to stress echocardiography (SE) results in patients with negative $(A)$ and positive $(B)$ exercise ECGs.

incremental information provided by stress echocardiography on survival analysis is shown in fig 3. Patients in whom both tests were positive had a significantly worse outcome, while no prognostic gain was found in patients with a negative exercise ECG.

\section{Discussion}

Although risk stratification after acute myocardial infarction is generally recommended, formal testing with the available diagnostic tools is limited by the ability of any test to predict which patients will subsequently have a clinical event. ${ }^{13}{ }^{14}$ Indeed, the predictive value of any test depends on the underlying event rate in the patient cohort. Patients recovering from uncomplicated acute myocardial infarction who are able to exercise have a very low pretest probability of hard events, ${ }^{2}$ so that only tests with almost absolute sensitivity and specificity could provide an effective stratification of risk. In the recent past, many studies have claimed to show an advantage of one specific stress test technique over another, generally by head to head comparisons in a given population. However, this approach is of limited clinical value as it does not assess the additional diagnostic or prognostic information provided by the test in question. Good clinical practice requires the physician to choose tests in a cost-effective manner, where each test, individually or sequentially, may lead to improved diagnosis and management. Moreover, test ordering is cost-effective if the physician integrates the results with the available clinical information, and a test is helpful only to the extent that it provides nonredundant information with potential impact on patient care. ${ }^{15}$
FINDINGS IN THIS STUDY

We found no clinical differences between patients who did or did not have spontaneous events during follow up, nor could spontaneous events be predicted in patients with a high threshold positive exercise ECG, although low threshold positive exercise electrocardiography was predictive. The addition of stress echocardiography resulted in much better discrimination in patients with a high threshold positive exercise ECG. Patients with a positive low threshold exercise ECG and positive stress echocardiography showed the highest event rate of all. These findings are of relevance for two main reasons: first, patients with uncomplicated acute myocardial infarction are known to have a small but definite risk of subsequent cardiac events, so prognostic stratification has important clinical and economic implications; second, the outcome of patients at increased risk appears to be improved by aggressive treatment. ${ }^{316}$

\section{COMPARISON WITH PREVIOUS STUDIES}

Only a few studies have specifically compared the prognostic value of exercise ECG with that of stress echocardiography after uncomplicated acute myocardial infarction. Greco and colleagues reported that dobutamine stress echocardiography was superior to exercise ECG, and independent and additive with respect to the standard clinical variables ${ }^{17}$; however, they found no difference between exercise ECG and dobutamine stress echocardiography in predicting death and unstable angina-only stress echocardiography was able to predict reinfarction. A drawback of that study was that the numbers were very small and there were few spontaneous events; in particular, only six reinfarctions were observed during 17 months of follow up.

By multivariate analysis dipyridamole stress echocardiography was more robust than clinical and exercise ECG variables in the EPIC (echo Persantine international cooperative) study. ${ }^{18}$ As in our study, the EPIC investigators found that a negative maximum exercise ECG identified a very low risk subgroup. However, although a stepwise prognostic algorithm similar to that employed in the current study was used, no stratification of exercise ECG positivity was provided in their study.

Additive prognostic value of the exercise ECG and dipyridamole stress echocardiography was reported by Sclavo and colleagues. ${ }^{19}$ In their series of 142 patients presenting with acute myocardial infarction and receiving thrombolysis, the combination of these two tests predicted spontaneous cardiac events with a sensitivity of $81 \%$ and a specificity of $60 \%$.

Our current study shows the incremental value of stress echocardiography as an adjunct to exercise ECG after uncomplicated acute myocardial infarction in a large group of patients with a long follow up. Despite its well known shortcomings, exercise ECG remains the first option for risk stratification in patients with uncomplicated acute myocardial infarction. ${ }^{20}$ Although patients who can exercise 
generally represent a low risk population, a multiparametric evaluation of the exercise stress test can yield more effective prognostic stratification. In particular, a low exercise capacity predicts an unfavourable prognosis independently of the result of test, ${ }^{21}$ while variables reflecting extension of the jeopardised myocardium - such as low threshold ECG ischaemia - are clearly associated with a worse outcome. ${ }^{22}$ On the other hand, the prognostic significance of high threshold ischaemia is much less definite. Our findings reinforce the prognostic impact of low threshold positive and maximum negative exercise ECG. Indeed, no substantial gain by imaging techniques can be expected in these patients upon Bayesian grounds. Conversely, a more than fivefold increase in risk was observed in patients with positive stress echocardiography in patients with high threshold positive exercise electrocardiography. This emphasises the advantage by the more sensitive mechanical signal at intermediate levels of pretest probability.

Stress echocardiography did not add prognostic value in patients with a negative stress ECG in our study. Possible explanations for this include a high prevalence of single vessel disease in such patients, the exclusion of patients with resting ECG abnormalities and with a low ejection fraction, and the end points chosen in our study. A previous study ${ }^{7}$ in which a higher negative predictive value of stress echocardiography was reported included patients with a greater degree of left ventricular dysfunction, in whom inducible ischaemia is likely to have more impact on prognosis; the study also had more composite end points, including ventricular arrhythmias and congestive heart failure. On the other hand, our results are in keeping with the report by Piccalò and associates on the very favourable outcome (97\% event-free survival for the combined end point of death and reinfarction) in patients with uncomplicated myocardial infarction and a negative predischarge exercise ECG. ${ }^{23}$ Under such circumstances it is unlikely that any further prognostic gain could be achieved by stress imaging.

\section{STUDY LIMITATIONS}

We presented pooled results of dipyridamole and dobutamine stress echocardiography in this study. Although these two agents induce ischaemia through different physiological mechanisms, they are known to have very similar diagnostic and prognostic accuracy. ${ }^{24} 25$

Coronary angiography was not included in this study, so no correlation was possible with coronary anatomy. However, the systematic recourse to coronary angiography in low risk subjects is not evidence based and does not comply with international guidelines.

Our results apply strictly to patients with uncomplicated infarction. Therefore they cannot be extrapolated to higher risk populations, such as patients with complicated infarction or a low ejection fraction. However, those populations generally undergo direct coronary angiography and eventual revascularisation, as a non-invasive risk stratification strategy is ex- pected to be neither rewarding nor costeffective. Conversely, patients with uncomplicated infarction have a low but definite risk of cardiac events and represent a puzzling challenge for the cardiologist. Present international guidelines recommend an ischaemia guided, non-invasive strategy for risk stratification; thus any attempt to improve the accuracy and costeffectiveness of this approach is of major interest in clinical practice.

\section{CLINICAL IMPLICATIONS}

The results of this study emphasise that the exercise ECG still represents the primary option for risk stratification, with pharmacological stress echocardiography as a valuable alternative where exercise ECG is inconclusive or not feasible. Patients with negative or low threshold positive exercise ECG have a well defined level of risk ${ }^{21}$ and no incremental information can be expected by imaging techniques. On the other hand, the definition of risk is much more difficult in patients with a high threshold positive exercise ECG. ${ }^{21}$ In such patients, pharmacological stress echocardiography contributes to risk stratification, allowing the physician to identify those in whom an aggressive strategy may be warranted.

1 Newby LK, Califf RM, Guerci A, et al. Early discharge in the thrombolytic era: an analysis of criteria for uncomplicated thrombolytic era: an analysis of criteria for uncomplicated
infarction from the global utilization of streptokinase and infarction from the global utilization of streptokinase and rt-PA for occluded coronary

2 Volpi A, De Vita C, Franzosi MG, et al. Determinants of 6 -month mortality in survivors of myocardial infarction after thrombolysis. Circulation 1993;88:416-29.

3 Madsen JK, Grande P, Saunamäki K, et al. Danish multicenter randomized study of invasive versus conservative treatment in patients with inducible ischemia after thrombolysis in acute myocardial infarction (DANAMI). Circulation 1997;96:748-55.

4 Boden WE, O'Rourke RA, Crawford $\mathrm{MH}$, et al. Outcomes in patients with acute non-Q-wave myocardial infarction randomly assigned to an invasive as compared with a conservative management strategy. Veterans affairs a conservative management strategy. Veterans affairs non-Qwave infarction strategies in hospital (VANQ
investigators. N Engl f Med 1998;338:1785-92.

5 Bolognese L, Sarasso G, Bongo AS, et al. Stress testing in Bolognese L, Sarasso G, Bongo AS, et al. Stress testing in
the period after infarction. Circulation 1991;83(suppl III):III-32-7.

6 Picano E, Landi P, Bolognese L, et al. Prognostic value of dipyridamole echocardiography early after uncomplicated myocardial infarction: a large-scale multicenter trial: EPIC Study. Am F Med 1993;95:608-10.

7 Carlos ME, Smart SC, Wynsen JC, et al. Dobutamine stress echocardiography for risk stratification after myocardial infarction. Circulation 1997;95:1402-10

8 Ryan T, Armstrong WF, O'Donnel JA, et al. Risk stratification after acute myocardial infarction by means of exercise two-dimensional echocardiography. Am Heart f 1987;114: 1305-16.

9 Marchioli R, Bomba E, Di Pasquale A, et al. A chart of postacute myocardial infarct risk: results in the first 18 months of the GISSI follow-up study. G Ital Cardiol 1998;28:416-33.

10 Schiller NB, Shah PM, Crawford M, et al. Recommendations for quantitation of the left ventricle by twotions for quantitation of the left ventricle by two-
dimensional echocardiography. If Am Soc Echocardiogr 1989;2:358-67.

11 Arnese M, Fioretti PM, Cornel JH, et al. Akinesis becoming dyskinesis during high-dose dobutamine stress echocardiography: a marker of myocardial ischemia or a mechanical phenomenon. Am f Cardiol 1994;73:896-9.

12 Zweig MH, Campbell G. Receiver-operating characteristic (ROC) plots: a fundamental evaluation tool in clinical medicine. Clin Chem 1993;39:561-77.

13 Moss AJ, Goldstein RE, Hall WJ, et al, for the Multicenter Myocardial Ischemia Research Group. Detection and significance of myocardial ischemia in stable patients after recovery from an acute coronary event. $¥ A M A$ 1993;269: 2379-85

14 Myers MG, Baigrie RS, Charlot ML, et al. Are routine noninvasive tests useful in prediction of outcome after myocar-
dial infarction in elderly people? Lancet 1993;342:1069-72.

15 Fineberg HV, Hiatt HH. Evaluation of medical practice. The case for technology assessment. $N$ Engl $f$ Med The case for
1979;301:1086.

16 Chaitman BR, McMahon RP, Terrin M, et al. Impact of treatment strategy on predischarge exercise test in the thrombolysis in myocardial infarction (TIMI) trial. Am $\mathcal{f}$ Cardiol 1993;71:131-8. 
17 Greco CA, Salustri A, Seccareccia F, et al. Prognostic value of dobutamine stress echocardiography early after uncomplicated myocardial infarction: a comparison with exercise electrocardiography. F Am Coll Cardiol 1997;29:261-7.

18 Picano E, Sicari R, Baroni M, et al. The relative value of exercise electrocardiography and dipyridamole stress echocardiography for risk stratification early after uncomplicated myocardial infarction. The EPIC (echo Persantine international cooperative) study group. G Ital Cardiol 1997, 27:32-9.

19 Sclavo M, Noussan P, Morselli L, et al. The usefulness of echo-dipyridamole and exercise tests in risk stratification in patients with acute uncomplicated myocardial infarct after systemic thrombolysis. Cardiologia 1991;36:357-62.

20 American College of Cardiology/American Heart Association. ACC/AHA guidelines for the management of patients with acute myocardial infarction. $7 \mathrm{Am}$ Coll Cardiol 1996;28:1328-428.

21 Villella A, Maggioni AP, Villella M, et al. Prognostic significance of maximal exercise testing after myocardial cance of maximal exercise testing after myocardial data-base. Lancet 1995;346:523-9.
22 Snader CE, Marwick TH, Pashkow FJ, et al. Importance of estimated functional capacity as a predictor of all-cause mortality among patients referred for exercise thallium single-photon emission computed tomography: report of 3400 patients from a single center. F Am Coll Cardiol 1997; 30:641-8.

23 Piccalò G, Pirelli S, Massa D, et al. Value of negative pre-discharge exercise testing in identifying patients at low risk after acute myocardial infarction treated with thromrisk after acute myocardial infarction

24 Minardi G, Di Segni M, Manzara CC, et al. Diagnostic and prognostic value of dipyridamole and dobutamine stress echocardiography in patients with Q-wave acute myocardial infarction. Am 7 Cardiol 1997;80:847-51.

25 Pingitore A, Picano E, Varga A, et al. Prognostic value of pharmacological stress echocardiography in patients with known or suspected coronary artery disease: a prospective, large scale, multicenter, head-to-head comparison between dipyridamole and dobutamine test. $\mathcal{F}$ Am Coll Cardiol 1999; 34:1769-77.

\section{IMAGES IN CARDIOLOGY}

\section{Exercise induced widening of the QRS complex in a patient on flecainide}

This case illustrates a toxic effect of flecainide in widening the QRS complex and inducing repolarisation abnormalities on exercise testing in a 67 year old woman, who had presented breathless in atrial fibrillation. Neither history nor examination revealed underlying cardiac abnormality; thyroid function and echocardiography were normal. The figure illustrates the electrocardiographic anterior chest leads during Bruce exercise tests. In the left half, the dose was $150 \mathrm{mg}$ twice daily: the first panel

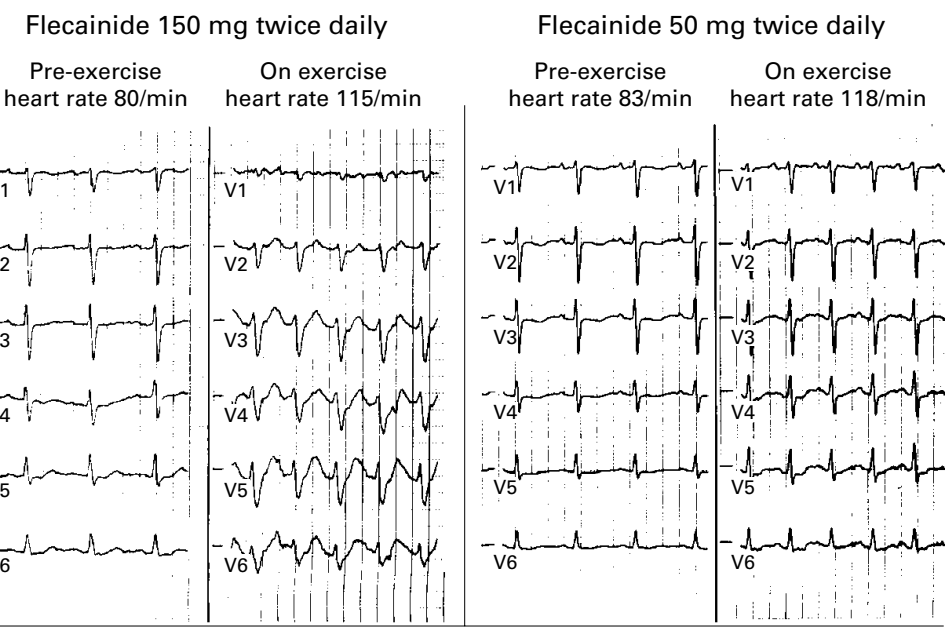

shows the pre-exercise trace, and the second panel stage II, heart rate 115 beats per minute. A dramatic widening of the QRS complex, increase in $\mathrm{T}$ wave amplitude, and prolongation of the QT interval occurred. This suggested pro-arrhythmic potential, and the dose was reduced to $50 \mathrm{mg}$ twice daily. The right half shows re-exercise, illustrating lack of the previous abnormalities (at a very similar exercise heart rate).

Flecainide caus es pronounced inhibition of the fast sodium channel, depressing the upstroke slope of the action potential, widening the QRS complex. Exercise augments the toxic effects of flecainide. The cardiac arrhythmia suppression trial (CAST) highlighted proarrhythmia, when patients post-myocardial infarction with ventricular ectopic beats treated with flecainide suffered greater mortality than placebo treated patients. The interaction of ischaemia and flecainide may be important in the mechanism for such increased mortality. There is general acceptance that flecainide is contraindicated in coronary patients, especially those with heart failure. Our case reminds us of these dangers, and suggests that exercise may assist in the assessment of proarrhythmic risk in patients treated with flecainide.

N TURNER

B C THWAITES bct.card@btinternet.com 